\title{
Forests at the limit: evolutionary - genetic consequences of environmental changes at the receding (xeric) edge of distribution. Report from a research workshop
}

\author{
Csaba MÁTYÁs ${ }^{1}$, Giovanni G. VENDRAMIN ${ }^{2}$, Bruno FADY ${ }^{3 *}$ \\ ${ }^{1}$ University of Western Hungary, Institute of Earth and Environmental Sciences, POB 132, 9401 Sopron, Hungary \\ ${ }^{2}$ CNR, Institute of Plant Genetics, Via Madonna del Piano 10, 50019 Sesto Fiorentino, Firenze, Italy \\ ${ }^{3}$ INRA, UR629, Ecologie des Forêts Méditerranéennes, Site Agroparc, 84914 Avignon Cedex, France
}

(Received 31 August 2009; accepted 21 September 2009)

In view of expected climatic changes, the adaptive potential of forest ecosystems and the future risks of diversity loss and extinction have received much attention. The behaviour of a species or of a population under changing conditions depends on its adaptive potential (genetic diversity, persistence, plasticity).

In ecology, judging of adaptability is based on the concept that the presence of a species (its distribution pattern) depends among other factors - on the limits of its physiological tolerance to climatic effects. This concept has to be extended, however, by the assertion that physiological tolerance is unquestionably determined by genetics. Limits of tolerance are therefore genetically set and will determine the presence or absence of a given species in a given environment. Thus, adaptive responses to environmental stress are to a large extent governed by genetic diversity, especially at the limits of distribution where strong shifts are expected.

Publications on shifts of vegetation triggered by climate change in the temperate belt are abundant. However, studies and analyses deal mostly with the shift of the "upper" or "front" thermal limits of distribution. Migration at the front or forward colonisation is the most visible and illustrative response to climate change. On the other hand, the retreat at the rear and xeric limit is insufficiently studied in spite of its ecological importance. Xeric species-margins may be defined as low altitude and low latitude limits of species distribution along a moisture balance gradient. In the northern hemisphere, xeric distribution limits of forests and forest tree species extend across the woodland ecotones of the Mediterranean, Southeast Europe, South Siberia and North America. The forest cover in these densely populated regions bears high ecological and social values; it is therefore an imperative to evaluate how available knowledge could help mitigate effects triggered by rapidly changing climate. At the xeric limit, selection pressure may narrow genetic variation and the increase in frequency of extreme events may result in growth decline and mortality.

What makes the problem of climatic selection at the xeric margin so special? Patterns and processes there are fuzzy, more difficult to follow than at the front limits. Human effects are omnipresent and ecological as well as socioeconomic consequences serious. Additional efforts are necessary to properly understand the heritable component of ecosystem responses to change, to mobilise the relevant research communities, and to make results understandable for stakeholders.

With the support of the European Network of Excellence "EVOLTREE", a research workshop was organised in Sopron (Hungary) by Prof. Csaba Mátyás (University of West Hungary) to provide a forum for discussions and the emergence of new concepts. The aim of the meeting was to present the state of knowledge and to identify future research needs with the objective of providing means to mitigate the effects of climatic selection pressure at the receding edge of forest tree species distribution. The intention of the organizer was to broaden both the geographic and disciplinary scopes of the topic to a global scale so as to raise awareness on the issue of receding, xeric limits of forests. The meeting was held in Sopron because it is located at the receding (xeric) edge of closed-canopy stand-forming temperate European forests.

Discussions covered three thematic areas:

1. Ecological, demographic and evolutionary perspectives of marginal and receding edge populations;

2. Genetic implications of extreme selection events: genomics of adaptation, genetic background of tolerance and plasticity;

3. Experiences from field trials, tasks for human intervention and research gaps at the receding edge of ranges.

\footnotetext{
* Corresponding author: bruno.fady@avignon.inra.fr
} 


\section{Conclusions and recommendations of the workshop}

\section{SOME DEFINITIONS ABOUT RANGE LIMITS}

Range limits of species follow the shift of environmental conditions. Receding limits appear at the rear of species ranges moving in one direction. Rear edge margins contain populations that have remained roughly in place for a very long time (usually in favourable topographic situations), at least since the Last Glacial Maximum, and their evolutionary trajectory has been largely independent of population dynamics across the main range. Trailing edge populations are left behind the contiguous range shift.

Xeric species margins may be defined as low altitude and low latitude limits of species distribution areas along a moisture balance gradient. Although determined primarily by climatic factors, xeric receding edges may also be influenced by interspecific interactions, edaphic conditions, anthropogenic effects, etc.

\section{XERIC RECEDING SPECIES MARGINS ARE PARTICULARLY AFFECTED BY CLIMATE CHANGE}

Receding edges, especially xeric limits are seldom addressed in the literature. This may come from the fact that they are not perceived as endangered because marginal populations often belong to widespread species. Also, no major shifts are noticeable there (although there are documented reports of increased dieback due to climate change and insect outbreaks triggered by increasing drought). Xeric range margins often occur in areas where species do not currently raise economic or conservation concerns. Although all climate models predict an increase in frequency, duration and intensity of drought, there is great uncertainty not only in species distribution models but also in the predicted climate scenarios themselves (effects of socio-economical and political decisions, lack of ecological variables, spatial resolution). This may have a disproportional impact on our ability to predict the fate of marginal populations. Current bioclimatic envelope models, set at species level are not accurate for determining the fate of xeric as well as of other edge populations.

\section{FUTURE RESEARCH DIRECTIONS FOR QUESTIONS THAT NEED URGENT ANSWERS}

\subsection{Where are xeric range margins?}

Current xeric range limits of species (external limits) are often not well defined and difficult to assess for at least four reasons: (1) Species range margins are ecologically very heterogeneous and fuzzy, especially where orography is complex (Mediterranean, Balkans) and soil conditions are very heterogeneous; (2) Low elevation/low latitude land has mostly been converted to agriculture, often leading to local extinction of marginal occurrences; (3) Forest management is often artificially influencing survival and regeneration, thus obscuring natural processes; (4) Lack of coordinated research. Without information on range margin limits, processes affecting local population persistence at range margins will not be properly understood. Delineating xeric range margins will also help improve climate envelope models.

\subsection{Can changes at the receding edge be predicted reliably?}

At ecosystem level, climate change alters within and among species competition and leads to manifold risks. The response of populations to extreme climate events at the xeric limit will depend on (i) species-specific responses set by the genetic system, and (ii) on difficult-to-forecast interactions and hazards, caused by known and/or newly emerging pests and diseases. To assess past and future changes at range limits and as a basis for modelling species and population responses to climate change, there is a pressing need for describing and monitoring (for example using remote sensing and permanent field plots) community level, spatial and age structures and reproductive success in receding edge populations.

\subsection{Are xeric edge populations valuable for conservation and future adaptation?}

Populations at range margins are often perceived as not valuable for conservation because they often display a lower genetic diversity. However, many ancient lineages have persisted in rear-edge populations. Because of selection and drift (among other factors), rear-edge populations may also contain divergent genetic resources and unique adaptations. Under climate change, sites of xeric edge populations will undergo strong ecological changes.

Extreme events may cause population extirpation and ancient lineages may disappear, along with their original genetic make-up. On the other hand, many populations display strong ecological resilience, possibly due to evolutionary history, mating system, epigenetics and phenotypic plasticity, or community dynamics. The contribution of all these processes needs to be better documented. Basic information on genetic (neutral and adaptive) and phenotypic diversity of populations at xeric range margins is still lacking.

\subsection{What are the adaptive processes involved at xeric range margins?}

Xeric margins are areas where populations face significant genetic adaptation challenges under any type of environmental change, but particularly under climate change. Several cutting edge tools and approaches are relevant to address adaptation 
at the xeric edge. New methods in molecular genetic and genomics, for example, allow for the identification of selection signatures and of clinal variations of adaptive traits. Associating marker loci, genotypes and related adaptive traits (e.g. genes related to stress tolerance, to phenology, to hydraulic conductance, etc.) is becoming possible. Association mapping is particularly promising for trees. Epigenetic processes should be better understood, as they could help explaining phenotypic plasticity, and can buffer maladaptive gene flow. Hybridization is another process that needs to be investigated in depth (e.g. in species complexes such as Quercus, Pinus, Abies, etc.) and may be exploited for genetic rescue. As a tool to understand adaptation, provenance tests and reciprocal transplants are very valuable. However, there is a need to add new tests that include marginal populations as well as marginal test sites and possibly also species mixtures. More generally, a better understanding of the relative importance of gene flow, drift, selection and genetic buffering (plasticity, epigenetics), at range margins as well as range-wide, is urgently needed.

\subsection{A combined genetic and ecological (demographic) approach is needed}

General models and investigations, combining demographic (mating system, seed production, dispersal, recruitment dynamics) and genetic processes, possibly set at the community level (e.g. including at least insects) and including population level information, are needed to describe, understand and then simulate receding edge population dynamics under climate change. Functionally meaningful phenotypic traits that are easy to monitor, need to be measured over large sample sizes and over long time.

\section{IMPLICATIONS FOR FOREST MANAGEMENT AND CONSERVATION}

Long-term management and conservation strategies will have to be modified. This includes principles of selection and maintenance of conservation areas, rules for the use of reproductive material (seed zone delineation, seed/plant transfer) and adjustment of silvicultural methods (regeneration techniques, regulation of stand structure) for managing ecosystems at the receding edge of distributions. Shifts triggered by current and future climate changes are occurring very fast. Active human support and yet-to-be-defined management strategies will be needed for conserving rear-edge ecosystems, including human-aided migration (introduction, transfer evacuation) of threatened populations. These alternative approaches to current management strategies, with all the uncertainties they may bear, need to be tested very soon.

\section{CONCLUSION}

Although they most probably contain original genetic combinations, rear-edge, xeric limit populations are poorly known and have received limited interest from scientists and forest managers alike. Much effort is needed to better understand and manage these populations which will prove critical for the benefits of economically and ecologically important forest species under climate change. 